\title{
Die Rot-Fichte (Picea abies), Baum des Jahres 2017
}

\author{
Veit Martin Dörken
}

\begin{abstract}
The red spruce (Picea abies) is the German tree of the year 2017. It is a frequently encountered timber tree in our forests and often planted as monocultures. Until fairly recently, the red spruce was the classic christmas tree in Germany. The various uses of the tree, along with its distribution, morphology, and biology are summarized here.
\end{abstract}

\section{Zusammenfassung}

Die Rot-Fichte (Picea abies) ist Baum des Jahres 2017. Da sie ein wichtiger Nutzholzbaum ist, wird sie in unseren Forsten bevorzugt gepflanzt, oft auch in Monokulturen. Früher war die Rot-Fichte auch der klassische Weihnachtsbaum. Neben verschiedenen Nutzungsmöglichkeiten werden Verbreitung, Morphologie und Biologie dieses Nadelbaumes vorgestellt.

\section{Einleitung}

Während in den vergangenen Jahren bei uns vor allem seltene heimische Baumarten zum Baum des Jahres gekürt wurden, fiel dieses Jahr die Wahl auf eine unserer häufigsten Baumarten, nämlich auf die Rot-Fichte. Neben der Rot-Fichte waren in vergangenen Jahren bereits drei weitere heimische Kieferngewächse Baum des Jahres: Weiß-Tanne
(2004), Wald-Kiefer (2007) und Europäische Lärche (2012). Mit der Ausrufung der Rot-Fichte zum 27. Baum des Jahres wurde eine der umstrittensten heimischen Baumarten ausgewählt. Die einen verbinden mit der Rot-Fichte eintönige, artenarme Monokulturen, andere hingegen einen der wichtigsten heimischen Forstbäume, dessen Holz vielfache Verwendung findet.

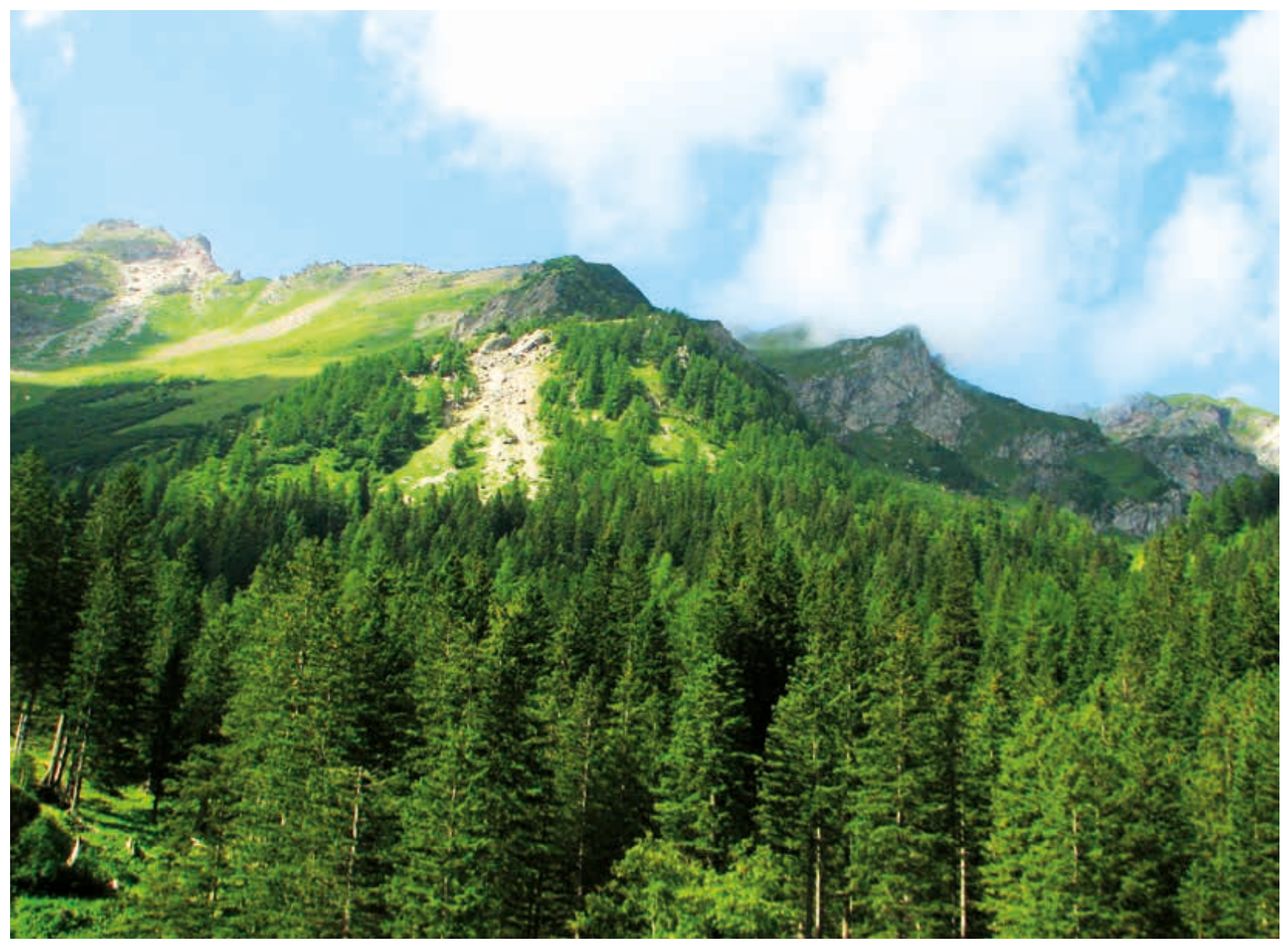

Abb. 1: Fichten-Bergwald in den Alpen. 


\section{Systematik \& Verbreitung}

Die Rot-Fichte (Picea abies) gehört zur Familie der Kieferngewächse (Pinaceae). In dieser Familie stehen neben der Kiefer (Pinus) auch Tanne (Abies) und Lärche (Larix). Innerhalb der Pinaceae gehören die Fichten zur Unterfamilie der Pinoideae, in der auch die Kiefer (Pinus) und die seltene Cathaya eingeordnet sind (FARJon 2010). Die Gattung Picea umfasst rund 40 Arten. Da besonders die ostasiatischen Fichten recht variabel sind, werden je nach Autor manchmal aber auch bis 80 Arten beschrieben.

Fichten kommen ausschließlich in den gemäßigten und kälteren Regionen der Nordhalbkugel vor. Ein Verbreitungsschwerpunkt ist Mittel- und West-China. In Europa sind nur Picea abies und P. omorika heimisch. Die Rot-Fichte ist eine nordost- bis mitteleuropäische Gebirgsbaumart, die in den Alpen bis in Höhen um $2200 \mathrm{~m}$ ü. NN vorkommt (Kiermeier 1993, Schütt et al. 2002). Dabei erstreckt sich ihr Areal von Mitteleuropa über die kontinental geprägten Gebiete Asiens bis nach Sachalin (SснÜтт et al. 2002). Die Rot-Fichte fehlte früher in vielen Gebieten, die niedriger als $600 \mathrm{~m}$ liegen (Kiermeier 1993). Aufgrund ihrer intensiven forstlichen Verwendung ist die Fichte mittlerweile fast in ganz Deutschland anzutreffen. Die Rot-Fichte kommt in unterschiedlichen Lebensbereichen vor. So ist sie in Nadel- und Laubmischwäldern, in schattigen Nordhangwäldern, aber auch auf Blockschutthalden und Felsfluren beheimatet. Auch am Rande von Hochmooren und in Auenwäldern ist sie anzutreffen, wobei die Standorte in Auenwäldern immer außerhalb des Überschwemmungsbereiches liegen (KIERMEIER 1993).

\section{Morphologie}

Die Rot-Fichte ist ein immergrüner Baum, der je nach Standort 20-40, seltener sogar 60 m Höhe und Stammdurchmesser bis 1,2 $\mathrm{m}$ erreichen kann. In der Jugend ist sie extrem starkwüchsig und kann durchaus einen jährlichen Längenzuwachs von einem Meter erreichen. Ab etwa $20 \mathrm{~m}$ Höhe verlangsamt sich das Wachstum deutlich (Mitchell 1979). Der Holzkörper ist nicht in Kern- und Splintholz gegliedert. Das Holz ist mit

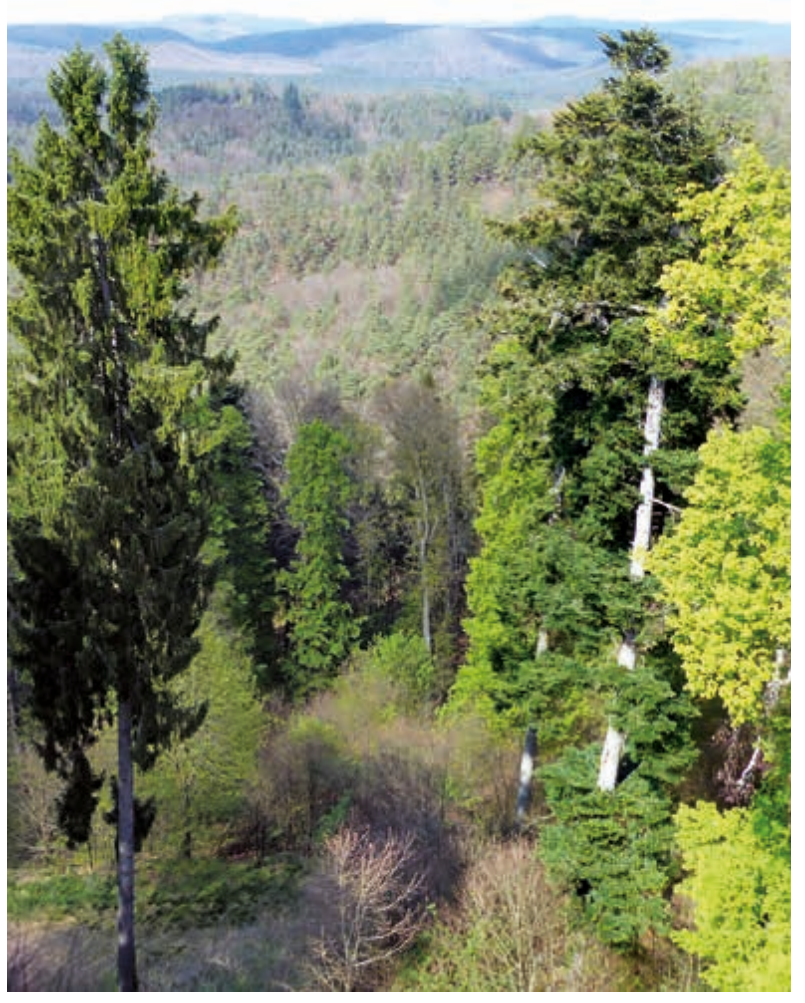

Abb. 2: Fichte (links) und Tanne (rechts) gehören zu den am höchsten werdenden Bäumen der heimischen Flora, hier ein Bestand in den Nord-Vogesen.

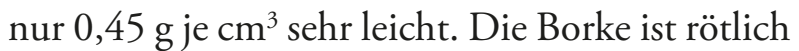
(Name Rot-Fichte!) und erst im hohen Alter grau gefärbt. Sie löst sich in mehr oder weniger großen, dünnen Platten vom Stamm ab und enthält bis $18 \%$ Gerbstoffe. Die breitkegelförmige Krone ist regelmäßig aufgebaut. Während die Bäume im Bestand meist nur in der Wipfelregion beastet sind, haben Exemplare in Solitärstellung bis unten Seitenäste. Die Hauptäste stehen quirlartig am Stamm verteilt und sind mehr oder weniger waagerecht orientiert mit meist aufstrebenden Triebspitzen. Eine Langtrieb-Kurztrieb-Differenzierung wie bei Lärche oder Kiefer fehlt. Aufgrund der hohen Variabilität der Kronenbeastung können drei verschiedene Beastungstypen unterschieden werden: Kammfichte (Äste hängend), Bürstenfichte (Äste hängend und abstehend) und Plattenfichte (Äste zweiter Ordnung vorwiegend abstehend, vgl. Sснüтt et al. 2002). Die Beastungstypen sind wahrscheinlich genetisch fixiert und können alle drei im gleichen Bestand gefunden werden (Sснӥтt et al. 2002). Die Zweige sind meist kahl, jedoch bei Bäumen aus den östli- 


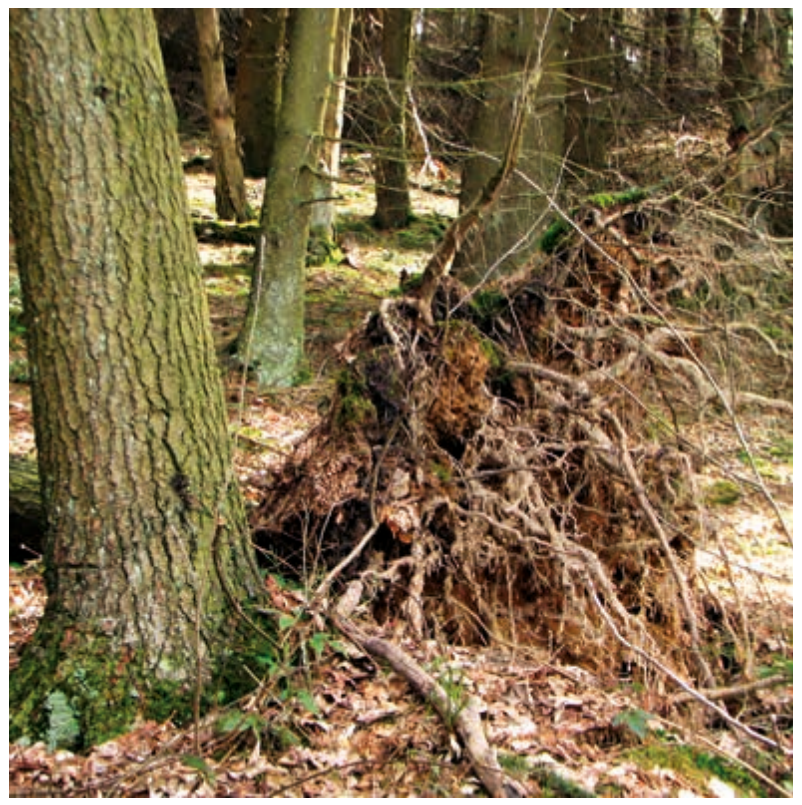

Abb. 3: Fichten sind Flachwurzler und können deshalb bei starkem Sturm leicht entwurzeln.

chen Provinzen des Verbreitungsareals häufig stark behaart (Mitchell 1977). Das weitstreichende Wurzelsystem ist ausgesprochen flach mit einem sehr hohen Anteil an Faserwurzeln im oberen Bodenhorizont sowie einigen Senkwurzeln.

Die braunen Knospenschuppen werden zum Zeitpunkt des Neuaustriebs haubenartig als Ganzes durch das Längenwachstum der Sprossachse abgerissen und auf den jungen Nadeln emporgehoben. Die Nadeln sind 1-3 cm lang mit einer stechenden Spitze („Die Rot-Fichte sticht, die Weiß-Tanne nicht"). Sie verbleiben 6-8 Jahre am Baum (Sснüтt et al. 2002). Die Nadeln stehen spiralig und sind kurz gestielt. Im Querschnitt sind sie vierkantig. Auf jeder der vier Nadelblattseiten sind deutliche Streifen vorhanden, in denen sich die Spaltöffnungen in mehreren Reihen befinden. Nach dem Abwurf bleiben immer Reste des Nadelblattstiels am Trieb erhalten (Unterschied zur Tanne), sodass sich die älteren, kahlen Äste rau anfühlen. Abgeworfene Nadeln sind schlecht abbaubar und tragen zur Bildung von mächtigen Rohhumusschichten und damit zur Bodenversauerung und Bodenverschlechterung bei.

Die Rot-Fichte ist einhäusig, d. h. Pollen- sowie Samenzapfen werden auf demselben Indivi-

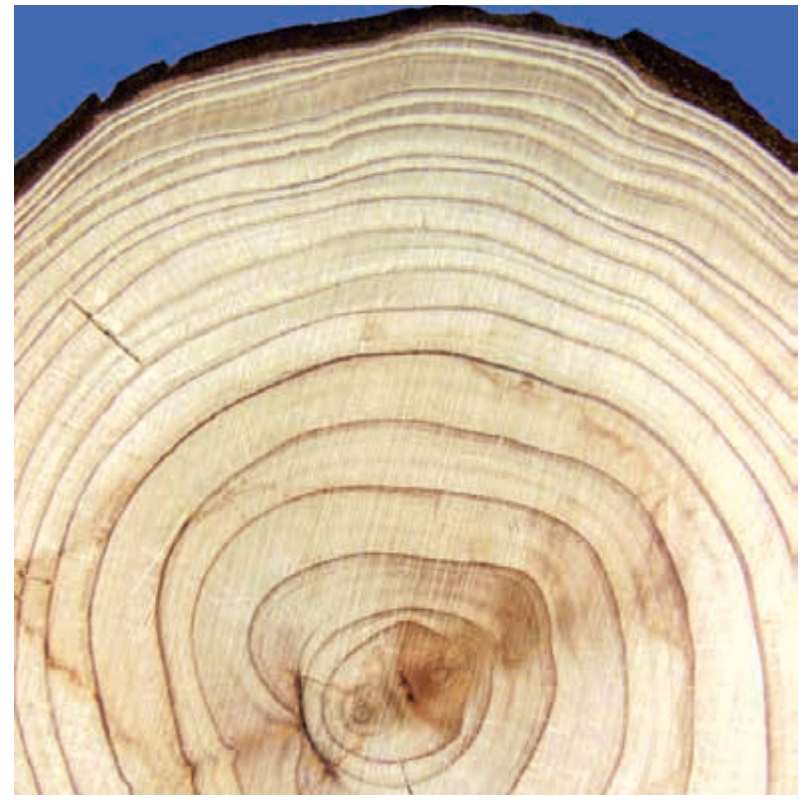

Abb. 4: Ausschnitt aus einer Fichtenholzscheibe mit ausgeprägten Jahresringen.

duum ausgebildet. Zur Verhinderung von Selbstbestäubung werden Samen- und Pollenzapfen auf dem Baum in unterschiedlichen Kronenregionen angelegt. Dabei stehen die Samenzapfen vor allem im Wipfelbereich, die Pollenzapfen bevorzugt in den unteren Kronenregionen. Die Rot-Fichte „blüht“ je nach Witterungsverlauf von Anfang April bis Mai.

Die Samenzapfen bestehen aus vielen Deck-/ Samenschuppen-Komplexen (vgl. Dörken \& JAGEL 2014). Zum Zeitpunkt der Bestäubung sind sie rötlich gefärbt und stehen aufrecht. Je Samenschuppe werden zwei Samenanlagen ausgebildet, deren Mikropylen und Bestäubungstropfen nach unten zeigen. Die Bestäubung erfolgt durch den Wind. Nach erfolgreicher Bestäubung ändern die Samenzapfen durch Wachstumsprozesse im Zapfenstiel aktiv ihre Position und wenden sich abwärts, um zur Reife die geflügelten Samen später besser entlassen zu können. Die reifen Samenzapfen öffnen sich nur bei trocken-warmer Witterung, um eine Ausbreitung der Samen durch den Wind besser zu gewährleisten. Da es sich um einen Quellungsvorgang handelt, ist das Öffnen und Schließen der Zapfen ein reversibler Vorgang. Der reife Zapfen ist hellbraun und 8-18 cm lang. Die Samenschuppen sind verholzt, dabei jedoch immer 


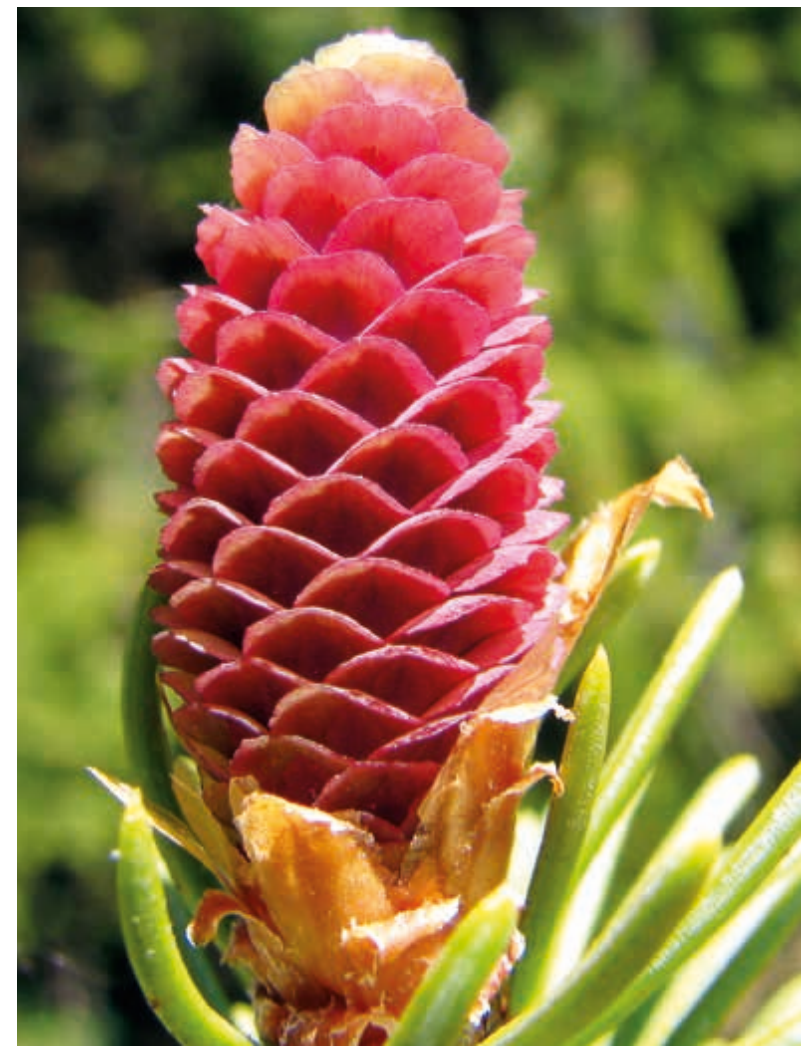

Abb. 5: Junger weiblicher Zapfen.

noch recht dünn. Sie haben einen ausgefransten bis gezähnten Rand. Die reifen Samenzapfen werden nach der Entlassung der Samen als Ganzes abgeworfen und zerfallen nicht. Umgangssprachlich werden am Boden liegende Fichtenzapfen häufig als Tannenzapfen bezeichnet. Tannenzapfen können es aber niemals sein, da diese während der Reife zerfallen.

Die männlichen, rötlichen Pollenzapfen stehen seitlich oder auf der Unterseite der Sprossachse. Die Pollenkörner haben zwei Luftsäcke. Diese sollen nicht unbedingt das Fliegen fördern, sondern das bojenartige Nachobendriften im Bestäubungstropfen zum Nucellus sicherstellen (Dörken \& Jagel 2014). Nach Entlassung des Pollens trocknen die Pollenzapfen rasch ein und fallen ab. Zur Zeit der Bestäubung wird oft so viel Pollen ausgestreut, dass er sich in Massen am Boden ansammelt und dann, wie auch bei der Kiefer (Pinus sylvestris), als Schwefelregen bezeichnet wird.

Die Samen der Rot-Fichte keimen im Frühjahr. Dabei ist der Bereich zwischen Wurzelhals und den Keimblättern im Vergleich zum Epikotyl recht

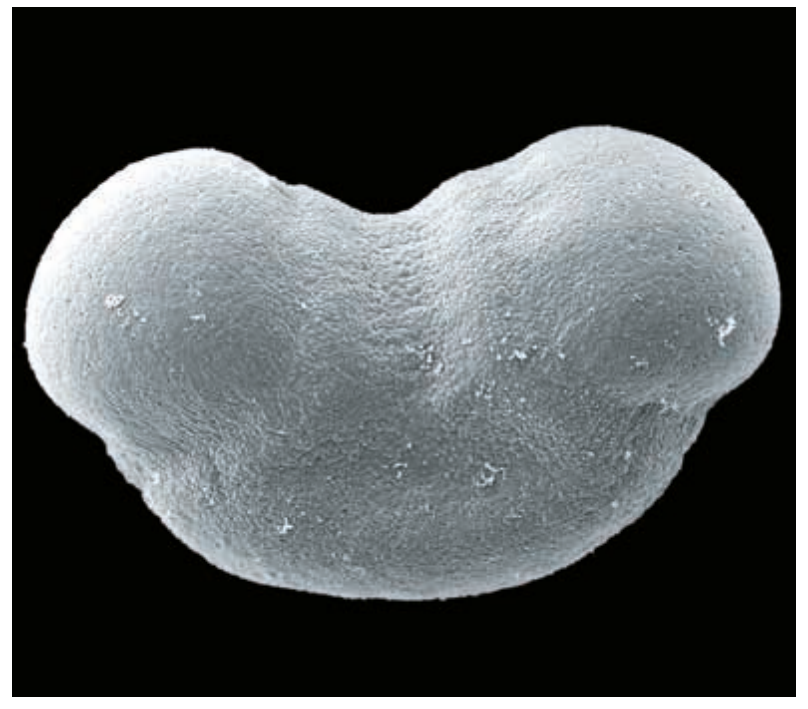

Abb. 6: Pollenkorn einer Fichte.

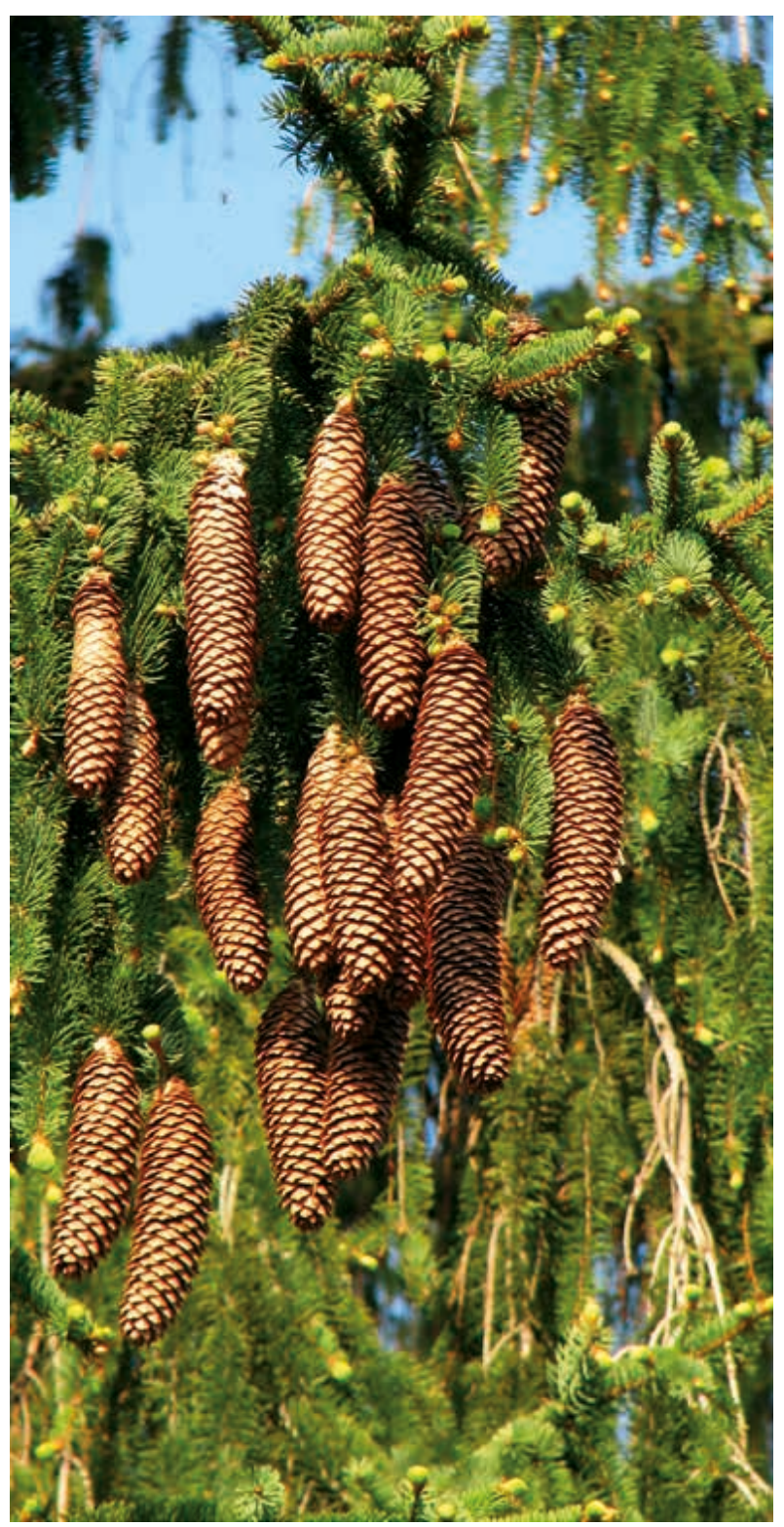

Abb. 7: Fichte mit reichlich Zapfen. 
lang. Die Anzahl der Keimblätter ist mit 5-10 sehr variabel.

\section{Eigenschaften}

Die Rot-Fichte braucht zum optimalen Gedeihen einen sonnigen bis halbschattigen Standort auf lockeren, durchlässigeren Böden. Bei starkem Schattendruck ist die Benadelung schütter. Je höher der Lehm- und Tongehalt, desto flacher wurzelnd und durch Windwurf gefährdeter sind die Bäume. Orkanstürme haben in deutschen Forsten schon manch eine Fichtenplantage umgeworfen. Die Sturmschäden des Orkans Kyrill, der im Januar 2007 über weite Teile Europas fegte, sind z. T. noch heute anhand der Kahlflächen oder jungen Aufforstungen im Sauerland zu erahnen. Dort waren großflächig Unmengen an Fichten innerhalb kurzer Zeit wie Streichhölzer umgefallen.

Generell reagiert die Rot-Fichte empfindlich auf Hitze- und Trockenstress, Boden- und Luftverschmutzungen. Auch gegenüber Salzbelastungen ist sie recht intolerant (Kiermeier 1993). Mit zunehmender Luftverschmutzung sinkt die Lebensdauer der Nadeln, sodass diese bei allzu hohen Rauchgasbelastungen die Nadeln nur sehr kurzlebig sind. Daher ist die Lebensdauer von Fichtennadeln ein wichtiger Bioindikator für den Grad der Luftverschmutzung.

\section{Verwendung}

Die Rot-Fichte ist eine der wichtigsten, leistungsfähigen heimischen Forstbäume, die ein vielseitig nutzbares, leichtes und helles Holz liefern, das sich gut als Bau- und Konstruktionsholz, aber auch zur Papierherstellung eignet. Auch im Möbelbau findet Fichtenholz vielfach Verwendung. Der Untertage-Abbau von Steinkohle an der Ruhr wäre ohne Fichtenholz als Stützen in seinen Anfängen nicht durchführbar gewesen. Das Harz dient zur Herstellung von Terpentin.

Fichtnadelöl sind ätherische Öle aus Trieben, Nadeln oder Zapfen von Fichten und anderen Nadelbäumen. Es hat beruhigende und bakterizide Wirkung. Es wird deshalb als Badezusatz, als Raumduft oder Inhalationsmittel verwendet.
Als Weihnachtsbaum ist die Rot-Fichte mittlerweile durch die Nordmann-Tanne verdrängt, denn sie duftet zwar sehr intensiv, verliert aber sehr schnell ihre Nadeln. Hochburg des Weihnachtbaumanbaus in Deutschland ist das Sauerland, wo es noch immer Rot-Fichten-Weihnachtsbaumplantagen gibt.

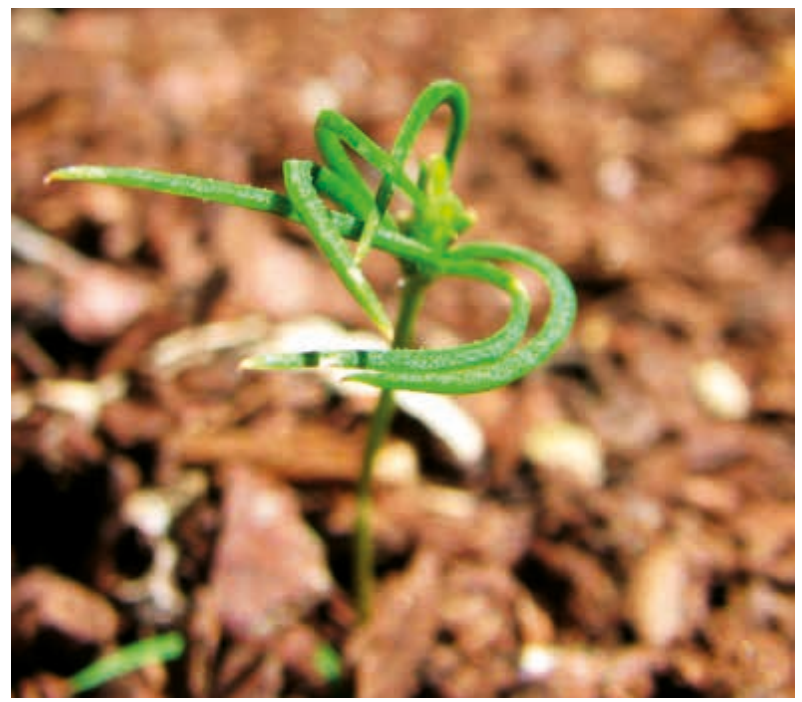

Abb. 8: Fichtenkeimling.

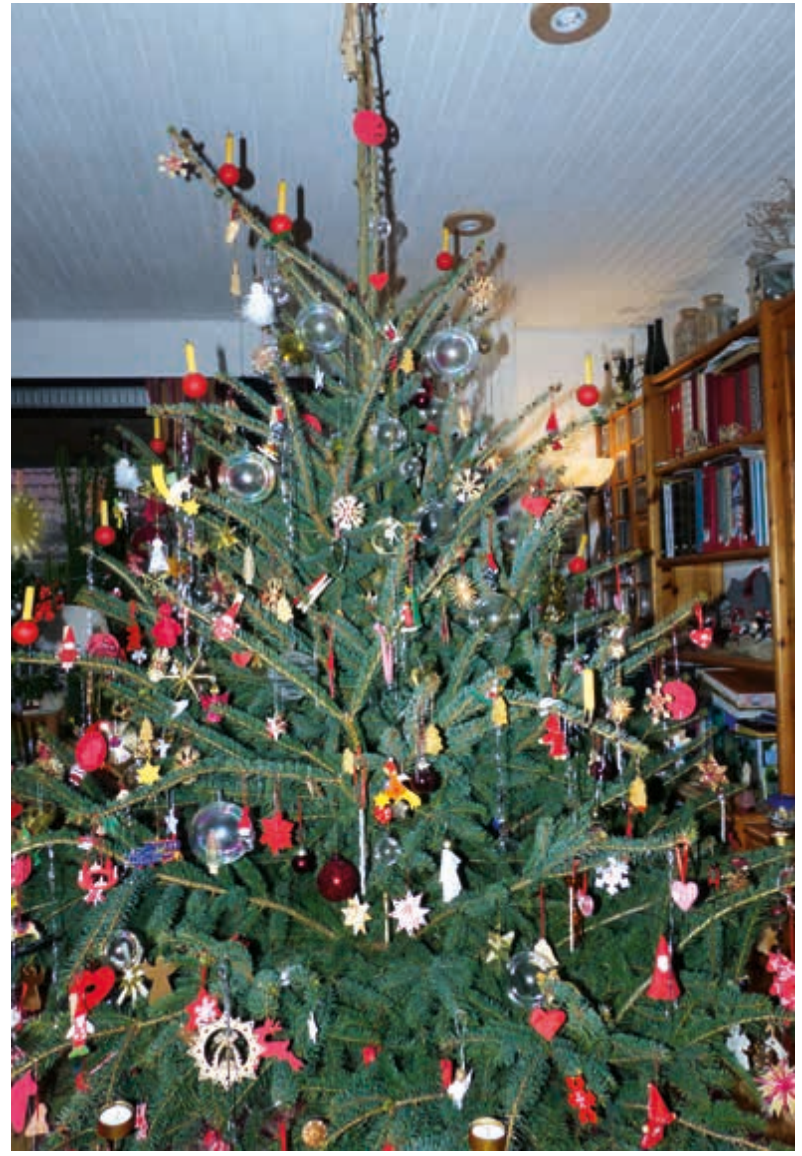

Abb. 9: Rot-Fichte als Weihnachtsbaum. 


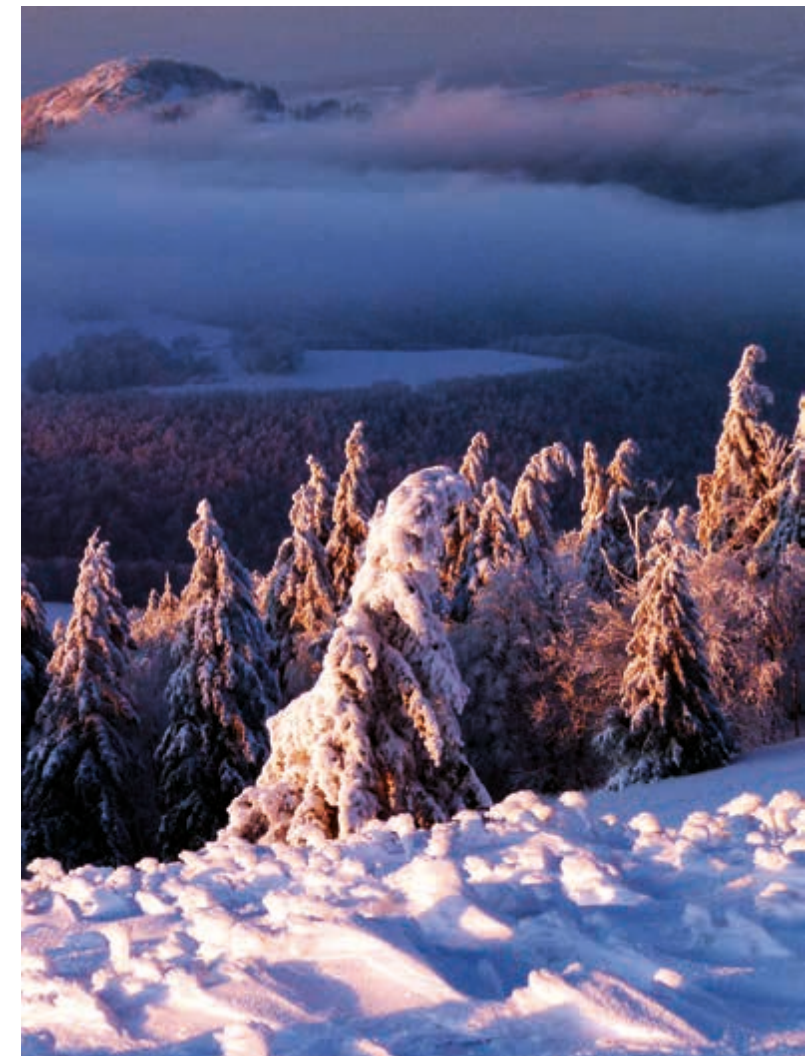

Abb. 10: Winternachmittag auf der Wasserkuppe in der Rhön mit verschneiten Fichten.

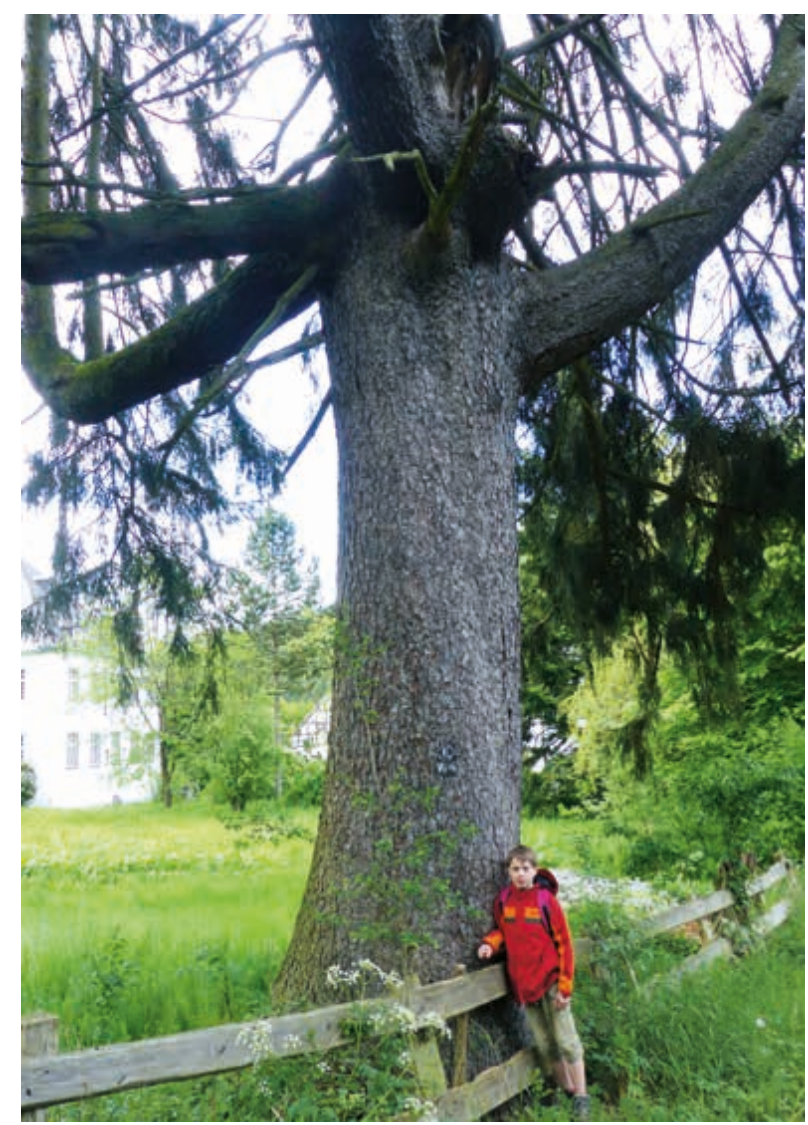

Abb. 11: Alte, große Fichte am Rittergut Blessenohl bei Wenholthausen im Sauerland.
Im Siedlungsbereich spielen besonders die gärtnerischen Selektionen eine wichtige Rolle als immergrüne Ziergehölze in Parkanlagen und Gärten. Hier werden jedoch anstelle der Wildart vielfach verschiedene, deutlich kleiner bleibende Sorten verwendet. Die aus Hexenbesen selektierten Zwergformen sind nicht nur in Steingärten, sondern auch zur Kübelbepflanzung geeignet. Bei der Verwendung als Heckenpflanze eignet sich die Rot-Fichte im Flachland nur bedingt, da sie dort recht kurzlebig ist und eine relativ schüttere Benadelung aufweist. Beliebte und interessante Sorten sind z. B. 'Arcocona' (unregelmäßig aufrechte Krone, bis $8 \mathrm{~m}$ hoch, bereits in jungen Jahren Zapfen bildend); 'Aurea' (Nadeln hellgelb, sonnenbrandgefährdet bei vollsonnigem Standort); 'Cupressina' (breite Säulenform, bis $20 \mathrm{~m}$ hoch); 'Echiniformis' (Zwergform, bis 0,3 m hoch werdend); 'Frohburg' (Hängeform); 'Inversa' (Hängeform, bis $8 \mathrm{~m}$ hoch werdend); 'Little Gem' (abgeflacht wachsende, bis $0,3-0,5 \mathrm{~m}$, extrem langsamwüchsige Zwergform mit schüsselartiger Vertiefung in der Kronenmitte); 'Nidiformis' (halbkugelige Zwergform, 2-3 m hoch); 'Procumbens' (Zwergform, teppichartig); 'Pumila Glauca' (niederliegende Zwergform mit auffälligen weißen Stomatastreifen an den Nadeln); 'Pygmaea' (sehr kompakte und dicht beastete, rundliche bis kugelige, 1-3 m hohe Zwergform); 'Virgata' (bizzarer, schmaler Habitus, wenige dicke und lange Ästen, mit wenig Seitentrieben, obere Äste zunächst noch aufrecht, die unteren hängend).

\section{Literatur}

Dörken, V. M. \& JAGEL, A. 2014: Orientation and withdrawal of pollination drops in the Cupressaceae s. 1. (Coniferales). - Flora 209: 34-44.

FARJON, A. 2010: A handbook of the world's conifers. Vol. I \& II. - Leiden.

Kiermeier, P. 1993: BdB-Handbuch, Teil VIII, Wildgehölze des mitteleuropäischen Raumes, 5. Aufl. - Pinneberg. Mitchell, A. 1979: Die Wald- und Parkbäume Europas, 2. Aufl. - Berlin.

Sснӥтt, P., Sснuск, H. J. \& Sтімm, B. 2002: Lexikon der Baum- und Straucharten. - Hamburg. 